\title{
Expression of arginase II in prostate cancer
}

\author{
SHANNON M. MUMENTHALER ${ }^{1}$, HONG YU ${ }^{1}$, SHEILA TZE ${ }^{1}$, STEPHEN D. CEDERBAUM ${ }^{2-4}$, \\ ANTHONY E. PEGG ${ }^{5}$, DAVID B. SELIGSON ${ }^{1}$ and WAYNE W. GRODY ${ }^{1,3,4}$ \\ Departments of ${ }^{1}$ Pathology and Laboratory Medicine, ${ }^{2}$ Psychiatry, ${ }^{3}$ Pediatrics, and ${ }^{4}$ Human Genetics, \\ David Geffen School of Medicine at UCLA, Los Angeles, CA; ${ }^{5}$ Department of Cellular \\ and Molecular Physiology, Pennsylvania State University, Hershey, PA, USA
}

Received September 24, 2007; Accepted November 2, 2007

\begin{abstract}
Previous reports have shown elevated arginase activity in prostate cancer patients. This study was designed to compare expression levels of arginase II (AII) in various human prostate cancer cell lines and tissues. Expression levels of AII and other enzymes involved in arginine metabolism were examined in androgen-dependent (LNCaP, LAPC-4) and androgen-independent (PC3, DU145, CL-1, CL-2) prostate cancer cell lines by real-time RT-PCR and Western blot analysis. Further expression analysis of AII was accomplished by immunohistochemical staining of a tissue microarray comprised of 246 primary prostatectomy specimens. In addition, polyamine levels were measured within the prostate cancer cell lines by HPLC. Total polyamines were significantly lower in the androgen-dependent cell lines compared to the androgen-independent cell lines. AII expression was found to be most prominent in the androgen-dependent cell lines and least prominent in the androgen-independent cell lines. Additionally, we found expression of ornithine aminotransferase (OAT), an enzyme also responsible for ornithine production, to be inversely correlated with AII expression. The tissue microarray data revealed that the highest AII expression was seen in BPH, followed by PIN and normal samples, with the lowest expression levels observed in prostate cancer tissues. Moreover, we observed an expression gradient across Gleason grades revealing stronger AII expression in low-grade tumors. The polyamine data, combined with the expression analysis studies, support a possible correlation between AII, OAT, and polyamine synthesis. Based on these results, arginase II expression may play a role in prostate cancer progression. More specifically, the elevated AII
\end{abstract}

Correspondence to: Dr Wayne W. Grody, Department of Pathology and Laboratory Medicine, David Geffen School of Medicine at UCLA, 650 Charles E. Young Drive, South, Box 951732, 1P-249 CHS, Los Angeles, CA 90095-1732, USA

E-mail: wgrody@mednet.ucla.edu

Key words: prostate cancer, arginase II, ornithine aminotransferase, tissue microarray, polyamines expression seen in androgen-dependent and in more differentiated prostate cancers suggests that AII could be a potentially useful marker of early stage prostate adenocarcinoma.

\section{Introduction}

Prostate cancer has the highest incidence rate of any cancer among men in the US with 230,000 new cases and 27,000 deaths reported annually (1). Despite its high prevalence, the heterogeneous nature of prostate cancer makes it difficult to understand the molecular mechanisms controlling benign and malignant prostatic cell growth. Currently, prostate-specific antigen (PSA) is the most common biochemical marker used to screen for prostate cancer; however, much controversy remains over its use due to the variability and uncertain predictive value observed (2). Therefore, it is important to continue to improve current diagnostic tools and determine new prognostic variables.

In addition, a better understanding of the biology of this common tumor is necessary if we are to improve both diagnosis and treatment. Polyamines play a crucial role in the development and progression of prostate adenocarcinoma (3). It is well known that the prostate and its secretions contain the highest levels of polyamines of any tissue, which are important for normal and neoplastic cell proliferation and differentiation (4-6). During the development of prostate cancer, polyamine ratios (spermidine:spermine), ornithine decarboxylase (ODC) activity, and the activities of other polyamine pathway enzymes are all elevated $(7,8)$. In the polyamine synthetic pathway, arginase produces ornithine, which is then available for conversion into polyamine compounds such as putrescine, spermidine, and spermine by ODC (9).

Arginine is an essential amino acid which feeds into many metabolic pathways that are important for normal cell growth and differentiation, including those for proline, glutamate, gamma-aminobutyric acid (GABA), nitric oxide, and polyamines (10-12). L-arginine is cleaved into ornithine and urea by arginase, which is the final enzyme in the urea cycle (13). There are two known isoforms of this enzyme, arginase I (AI) and arginase II (AII). The AI enzyme is localized in the cytosol and is involved in the detoxification of ammonia as part of the urea cycle. The AII enzyme, found in the mitochondria, is involved in the biosynthesis of 
polyamines, glutamate and proline, and the modulation of nitric oxide synthesis (iNOS) (14). A number of reports maintain that ODC is the initial enzyme in mammalian cell polyamine biosynthesis; however, very little is known in this regard about arginase, the enzyme that precedes ODC and produces ornithine $(15,16)$. Arginase II is expressed at high levels in the normal prostate, kidney, and brain and at low levels in the gastrointestinal tract (17). Elevated arginase activity has been found in patients with prostate, breast, lung, and colon cancer and the increased enzyme activity may be responsible for maintaining the necessary level of polyamines for tumor growth and development (14,18-20).

Despite reports of high arginase expression in the normal prostate and elevated arginase activity in prostate cancer patients, a comprehensive study comparing the expression levels of arginase II in prostate cancer cell lines, as well as in benign and malignant prostatic tissues, has not been performed. In the present study, we investigated the expression of AII in prostate cancer using both cell culture and tissue microarray (TMA) techniques (21). Specifically, we observed elevated AII expression in the androgen-dependent prostate cancer cell lines and in more differentiated tissues, suggesting that high AII expression may be an important marker for early-stage prostatic carcinoma. We have come to appreciate that arginine and its immediate metabolic products lie at the center of an important biochemical nexus, acted upon by a number of complementary and competing enzymes, including arginase II, ornithine decarboxylase, and ornithine aminotransferase (OAT), an enzyme responsible for synthesizing ornithine from pyrroline-5-carboxylate (P5C). With this in mind, we extended our cell culture studies to include this larger family of enzymes and found that OAT may be compensating for the loss of AII in undifferentiated prostate cancer by providing an alternative source of ornithine. Furthermore, polyamine analysis among the prostate cancer cell lines supports a possible correlation between AII, OAT, and polyamine levels. We postulate that the role of arginase II in prostate cancer is dependent upon its involvement in polyamine synthesis and its interaction with related enzymes in the arginine pathway.

\section{Materials and methods}

Cell culture. $\mathrm{LNCaP}, \mathrm{PC} 3$, and DU145 cell lines were acquired from ATCC (Manassas, VA) and cultured in RPMI-1640 medium supplemented with $10 \%$ FBS. The LAPC-4 cell line was obtained from Dr Charles Sawyers (UCLA, Los Angeles, $\mathrm{CA}$ and Sloan-Kettering Cancer Center, New York, NY) and maintained in Iscove's modified medium with $10 \%$ FBS. The LNCaP-derived androgen-independent clones, CL-1 and CL-2, were a gift from Dr Arie Belldegrun (UCLA) and were generated by growing LNCaP cells in androgen-free medium for 6 weeks followed by selection of the androgen-independent clones. After selection, the CL-1 cell line was maintained in RPMI-1640 medium with 10\% charcoal-stripped FBS (Hyclone, Logan, UT), while its counterpart, CL-2, was returned to RPMI-1640 medium with $10 \%$ FBS (22).

Quantitative real-time $R T-P C R$. Total RNA was extracted from human prostate cancer cells using the GenElute
Mammalian Total RNA miniprep kit (Sigma, St. Louis, MO). cDNA synthesis was performed on $1 \mu \mathrm{g}$ of total RNA using the iScript cDNA synthesis kit (Bio-Rad, Hercules, CA). Real-time PCR for arginase II (AII), ornithine aminotransferase (OAT), and ornithine decarboxylase (ODC) was performed on an iQ Real-time PCR detection system (Bio-Rad) using 96-well microplates. The reaction mixture contained $2 \mathrm{X}$ iQ SYBR Green supermix (Bio-Rad), forward and reverse primers, cDNA, and sterile water. Each sample was diluted 1:10 and the PCR efficiency for each primer was determined using a standard curve generated from cDNA serial dilutions. The following primer pair sequences were used for real-time PCR: Arginase II: 5'-CGCGAGTGCATTCCATCCT-3' and 5'-CCTTTTCATCAAGCCAGCTTCTC-3'; Ornithine aminotransferase: 5'-GACTGCCTGTAAACTAGCTCG-3' and 5'-AACGTCCTACCCCAGAAGTTC-3'; Ornithine decarboxylase: 5'-TGAAATACAGTTGGTGCAGAGTC-3' and 5'-ACTTTGCTTTGGGATGTGCTC-3'; GAPDH: 5'-CCT CAAGATCATCAGCAATGCCTCCT-3' and 5'-GGTCATG AGTCCTTCCACGATACCAA-3'. The real-time RT-PCR protocol was $95^{\circ} \mathrm{C}$ for $3 \mathrm{~min}$; 40 cycles of $95^{\circ} \mathrm{C}$ for $15 \mathrm{sec}$, $61^{\circ} \mathrm{C}$ for $30 \mathrm{sec}, 72^{\circ} \mathrm{C}$ for $30 \mathrm{sec} ; 95^{\circ} \mathrm{C}$ for $1 \mathrm{~min} ; 55^{\circ} \mathrm{C}$ for $1 \mathrm{~min} ; 80$ cycles of $55^{\circ} \mathrm{C}$ with increase in temperature by 0.5 every $10 \mathrm{sec}$ for melt curve. The expression level of each gene was normalized to GAPDH mRNA and the results were analyzed using the Gene expression macro analysis software (Bio-Rad). The data are presented as fold change in expression relative to the other cell lines in that particular experiment with the lowest expression given a value of 1 .

Western blot analysis. Cells were lysed in RIPA buffer with $1 \mathrm{X}$ Halt protease inhibitor cocktail at $4^{\circ} \mathrm{C}$ (Pierce, Rockford, IL). Protein concentrations were determined using the Bio-Rad protein assay. The cell lysates (40 $\mu \mathrm{g}$ protein) were loaded onto a $10 \%$ Tris- $\mathrm{HCl}$ ready gel (Bio-Rad) and transferred to PVDF membrane. The rabbit anti-arginase II antibody (1:400) purchased from Santa Cruz Biotechnology, Inc. (sc-20151, Santa Cruz, CA) is specific for arginase II detection and has no apparent cross-reactivity with the liver isozyme, arginase I. The mouse anti-GAPDH antibody (1:2500) was purchased from Imgenex (IMG-5019A-1, San Diego, CA) and the rabbit anti-OAT antibody (1:500) was a generous gift from Dr David Valle (Johns Hopkins University, Baltimore, MD).

Polyamine analysis of prostate cells. Prostate cancer cells were harvested, extracted, and deproteinized with $10 \%$ (w/v) trichloroacetic acid (TCA) when they were $80 \%$ confluent. Samples were then analyzed using an ion pair reversed-phase HPLC separation method and post-column derivatization with o-phthalaldehyde (23). Putrescine, spermidine, and spermine levels were determined for each prostate cancer cell line. The results from at least 3 different experiments per cell line were averaged and expressed as nmoles/mg of protein \pm standard deviation.

Prostate tissue microarray. Formalin-fixed paraffin-embedded tissue from primary radical prostatectomy cases between 1984 and 1995 (UCLA Medical Center) were randomly selected to use in the construction of this tissue microarray under IRB approval (24). It is comprised of 246 prostatectomy 


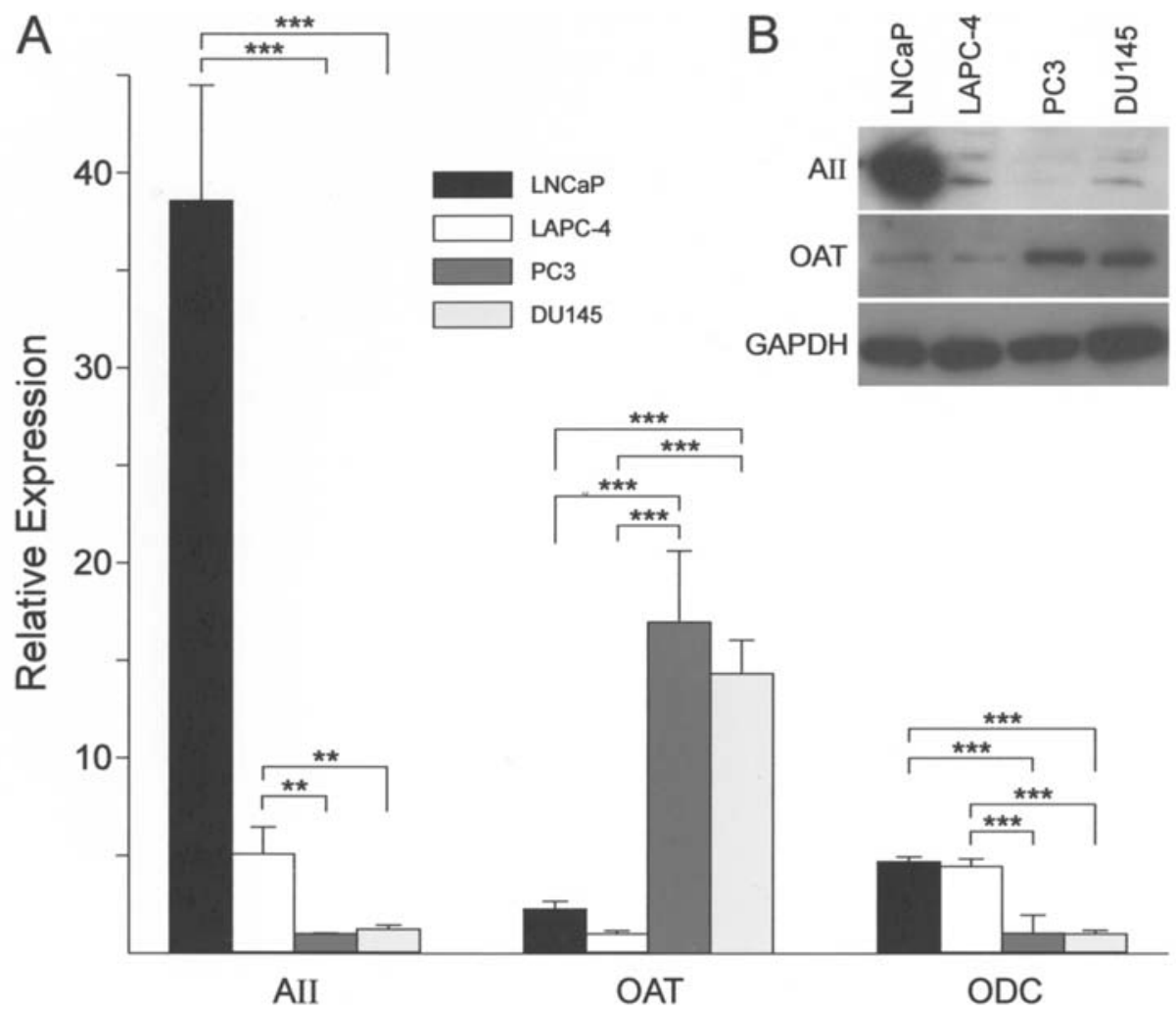

Figure 1. Expression analysis of selected arginine and polyamine metabolic genes in prostate cancer cell lines. (A) Quantitative real-time RT-PCR analysis of arginase II (AII), ornithine aminotransferase (OAT), and ornithine decarboxylase (ODC) in LNCaP, LAPC-4, PC3, and DU145 prostate cancer cell lines. Expression of each gene was normalized to the reference gene, GAPDH. The cell line with the lowest expression level for a particular gene was given a value of 1. LNCaP and LAPC-4 cells displayed higher levels of AII and ODC compared to PC3 and DU145 cells. In contrast, PC3 and DU145 cells exhibited higher levels of OAT than LNCaP and LAPC-4 cells. The vertical lines of the bracket indicate the two cell lines being compared to each other; ${ }^{*} \mathrm{p}<0.05$, ${ }^{* *} \mathrm{p}<0.01,{ }^{* * *} \mathrm{p}<0.001$. (B) Western blot analysis of AII and OAT in the LNCaP, LAPC-4, PC3, and DU145 cell lines. GAPDH protein was used as a lane loading control.

specimens that were embedded into 3 paraffin blocks, resulting in a total of 1,364 individual spots using the method of Kononen et al (21). At least 3 replicate tumor samples and one matching normal sample were taken from representative donor tissue. When available, matching benign prostatic hyperplasia $(\mathrm{BPH})$ and prostatic intraepithelial neoplasia (PIN) lesions accompanied the tumor spots. Staining with antiarginase II antibody generated 999 (73\%) informative tissue spots for analysis, including 251 normal, 109 BPH, 41 PIN, and 598 primary prostate cancer spots. In addition, a Gleason grade score of 1-5 was assigned to each prostate tumor spot (25). There was a total of 97 grade 1-2 (referred to as 'grade 2'); 337, grade 3; 139, grade 4; and 25, grade 5 spots.

Immunohistochemistry and scoring. A standard indirect immunoperoxidase staining method (R.T.U. Vectastain universal elite ABC kit, Vector Laboratories, Burlingame, CA) was used. Tissue array sections $(4-\mu \mathrm{m})$ were heated at $56^{\circ} \mathrm{C}$ for $30 \mathrm{~min}$ and then deparaffinized with xylene overnight. The following day, sections were rehydrated in graded ethanol. Antigen retrieval was performed using $0.01 \mathrm{M}$ sodium citrate buffer $(\mathrm{pH} 6.0)$ for $10 \mathrm{~min}$ at $95^{\circ} \mathrm{C}$. Endogenous peroxidase activity was quenched using $0.3 \%$ hydrogen peroxide in methanol for $30 \mathrm{~min}$ at room temperature. Consecutive addition of avidin D and biotin was performed as a blocking step prior to the addition of primary antibody (Avidin/Biotin blocking system, Vector Laboratories). Primary rabbit anti-arginase II polyclonal antibody was applied at a 1:400 dilution $(1 \mu \mathrm{g} / \mathrm{ml})$ for $1 \mathrm{~h}$ at room temperature (Santa Cruz Biotechnology, Inc.). For the negative control, non-immune rabbit $\operatorname{IgG}(1 \mu \mathrm{g} / \mathrm{ml})$ replaced the primary antibody. Staining was detected using diaminobenzidine (DAB) followed by Mayer's hematoxylin solution as the counter-stain. Sections were dehydrated and then coverslipped.

Scoring of the TMA IHC was performed by a pathologist (H.Y.). Semi-quantitative assessment of antibody staining was done in a blinded fashion. Only the prostatic glandular epithelium was scored. When staining was at minimum weak, but clearly visible, the stain would be considered positive. The proportion of positive cells $(0-100 \%)$ within the histology of interest was determined for each TMA spot and expressed as mean \pm standard error (26).

Statistical analysis. An unpaired Student's t-test was used to determine statistical significance in the cell culture and tissue based studies. For the tissue microarray data, associations between arginase II expression and pathological grade, stage, preoperative PSA level, and recurrence status were tested using the Pearson Chi-square test and the Kurskal-Wallis test (and Spearman correlation). Statistical analyses were carried out using the software package R (http://www.r-project.org). For all analyses, $\mathrm{p}<0.05$ was considered statistically significant and designated as ${ }^{*} \mathrm{p}<0.05,{ }^{* *} \mathrm{p}<0.01,{ }^{* * *} \mathrm{p}<0.001$ in the figures. 


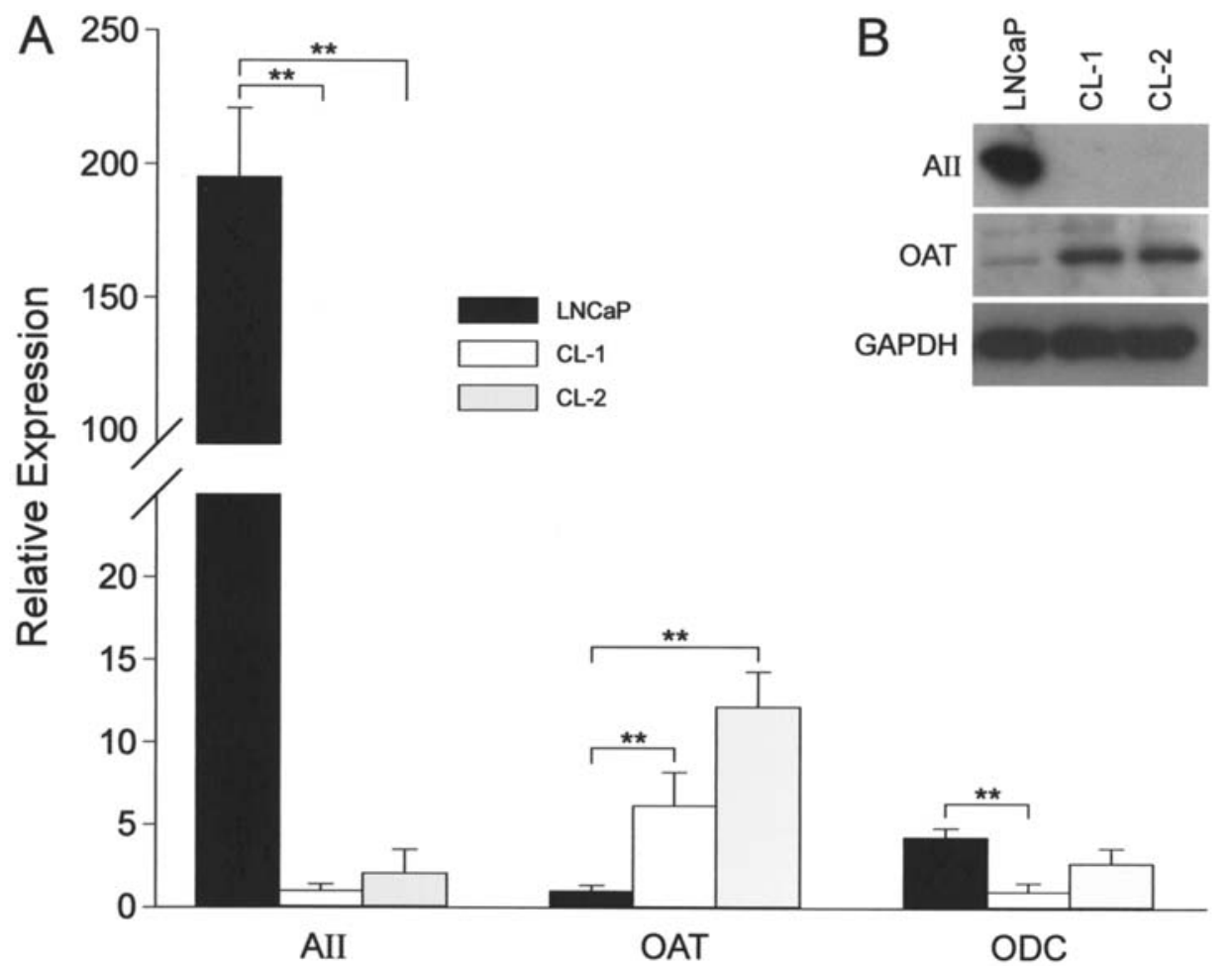

Figure 2. Expression analysis of selected arginine and polyamine metabolic genes in prostate cancer cell lines. (A) Quantitative real-time RT-PCR analysis of AII, OAT, and ODC in LNCaP, CL-1, and CL-2 prostate cancer cell lines. Expression of each gene was normalized to the reference gene, GAPDH. The cell line with the lowest expression level for a particular gene was given a value of 1. LNCaP cells displayed higher levels of AII compared to CL-1 and CL-2 cells. In contrast, CL-1 and CL-2 cells exhibited higher levels of OAT than LNCaP cells. The vertical lines of the bracket indicate the two cell lines being compared to each other; ${ }^{*} \mathrm{p}<0.05,{ }^{* *} \mathrm{p}<0.01,{ }^{* * *} \mathrm{p}<0.001$. (B) Western blot analysis of AII and OAT in the LNCaP, CL-1, and CL-2 cell lines. GAPDH protein was used as a lane loading control.

\section{Results}

Expression of arginase II and related enzymes in prostate cancer cells. Arginase II mRNA levels have been shown to be at least 10 times higher in the normal prostate than in all other normal tissues in which it is present (17). This result prompted us to examine AII expression in several established prostate cancer cell lines representing the extremes of differentiation and dedifferentiation, including LNCaP, LAPC-4, PC3, and DU145. Both the LNCaP and LAPC-4 cell lines are more differentiated and express PSA and androgen receptor $(27,28)$, while the PC3 and DU145 cell lines are less differentiated, do not express PSA, and are androgen-independent. When we examined AII protein levels by Western blot in these cell lines, we observed that AII expression was highest in the more differentiated, androgen-dependent prostate cancer cell lines, LNCaP and LAPC-4, with lower expression in the more undifferentiated and androgen-independent cancer cells, PC3 and DU145 (Fig. 1B).

In addition to examining AII expression, we extended our study to include OAT and ODC and performed quantitative real-time RT-PCR to assay the expression levels of these related genes in LNCaP, LAPC-4, PC3, and DU145 prostate cancer cell lines (Fig. 1A). Elevated RNA levels of AII and ODC were observed in the androgen-dependent cell lines, LNCaP and LAPC-4, with the lowest levels seen in the androgen-independent cell lines, PC3 and DU145. In contrast, OAT levels were lowest in LNCaP and LAPC-4 cells and the highest expression was observed in PC3 and DU145 cells.
The differential expression of AII among the prostate cancer cell lines was the most prominent of all the genes. From the real-time RT-PCR data, we observed an inverse relationship between AII and OAT levels among the cell lines. Western blot analysis of AII and OAT in LNCaP, LAPC-4, PC3, and DU145 cell lines confirmed this finding (Fig. 1B). It appears that when AII expression is high in the androgen-dependent cell lines, OAT levels are low. The opposite trend is seen in the androgen-independent cell lines which have high OAT expression and very low AII expression.

Differential expression of AII in androgen-dependent versus androgen-independent cell lines. To further explore whether arginase II is differentially expressed among androgendependent versus androgen-independent prostate cancer cell lines, we compared AII expression between LNCaP, CL-1, and CL-2 cell lines. As mentioned previously, CL-1 and CL-2 were created by growing $\mathrm{LNCaP}$ cells in androgen-depleted media for 6 weeks followed by selection of androgenindependent clones. The main difference between the two clones is CL-1 has been continually maintained in androgendepleted media, whereas CL-2 was returned to androgencontaining media after the 6 weeks of selection. It was also determined that both clones were PSA-negative and androgen receptor (AR)-negative (22). The LNCaP-derived androgenindependent clones CL-1 and CL-2 showed a loss in arginase II RNA and protein levels compared to the original LNCaP cell line (Fig. 2A and B, respectively). Essentially, AII is differentially expressed in androgen-dependent versus 

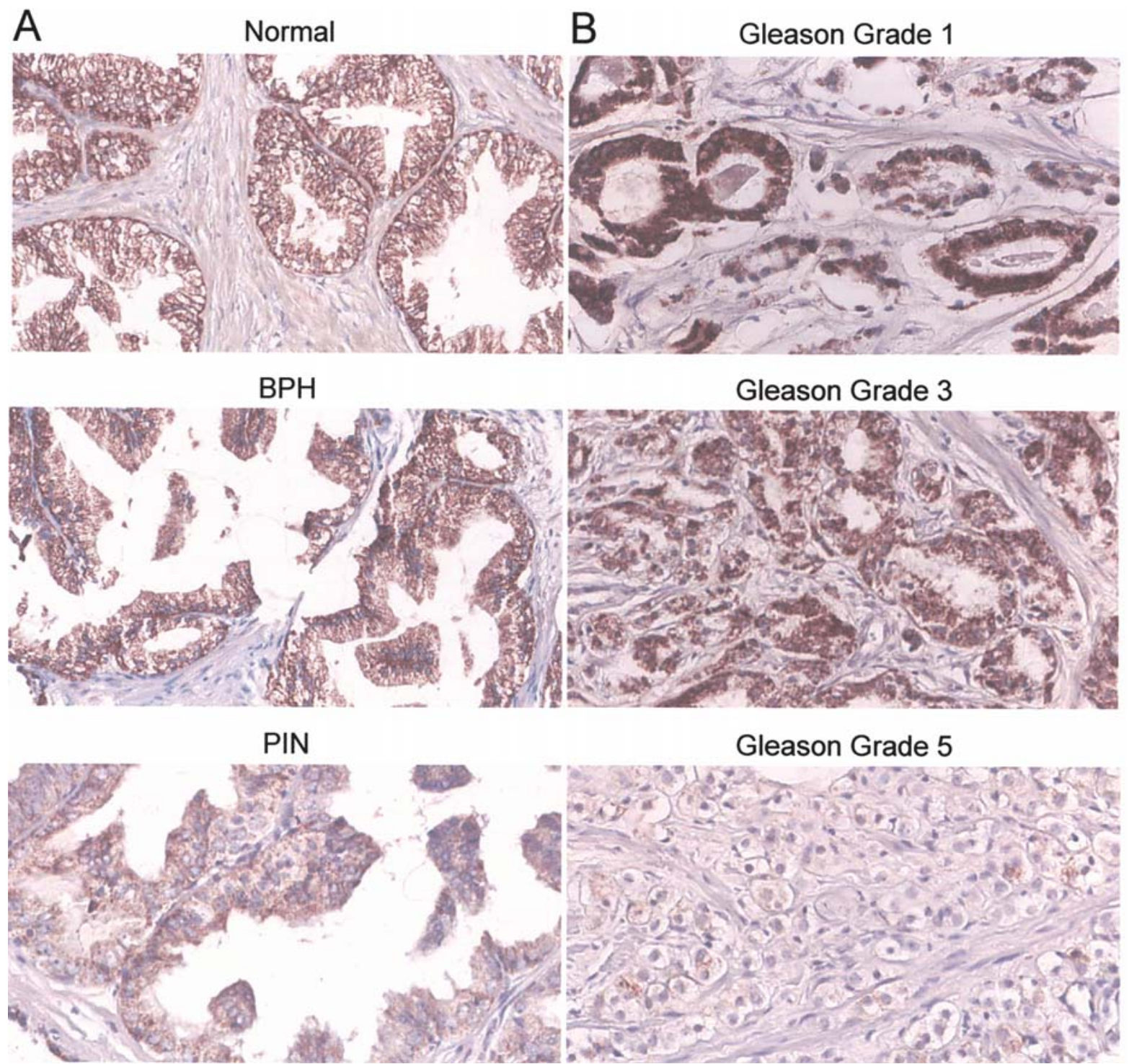

Figure 3. Immunohistochemical detection of arginase II expression in the glandular epithelial region of human prostate tissue samples within a tissue microarray. (A) From top to bottom: normal tissue spot, benign prostatic hyperplasia (BPH) tissue spot, and prostatic intraepithelial neoplasia (PIN) tissue spot. Normal, BPH, and PIN at a magnification of x20. (B) From top to bottom: low-grade tumor spot (Gleason grade 1), intermediate grade tumor spot (Gleason grade 3), and high-grade tumor spot (Gleason grade 5). Gleason grade 1 and Gleason grade 3 at a magnification of x20. Gleason grade 5 at a magnification of $\mathrm{x} 10$.

androgen-independent prostate cancer cell lines. In addition, OAT levels were increased in the CL-1 and CL-2 cell lines with respect to $\mathrm{LNCaP}$, while ODC levels were decreased in the LNCaP-derived androgen-independent clones. CL-1 and CL-2 cells behave more similarly to the androgen-independent cell lines, PC3 and DU145, with respect to AII and OAT expression than to the cell line they originated from, LNCaP.

Arginase II expression in prostate tissue sections. In conjunction with the previously mentioned in vitro experiments, we also examined arginase II expression in patient-derived prostatic carcinoma tissues. Among our initial sampling of individual tissues $(n=6)$, we observed greater AII staining intensity among prostate tumor sections compared to normal prostate tissue. Bronte et al performed immunohistochemistry (IHC) on a small number of individual normal $(n=2)$ and cancerous prostate tissues $(n=4)$ and also detected higher AII expression in prostate cancer versus tumor-free prostatic tissue (29). Additionally, we noted a gradient of AII expression across the various prostate cancer tumor grades with higher AII expression seen in the lowest Gleason grade tumors (data not shown). This result led us to conduct a highthroughput analysis of arginase II expression by immunohistochemistry on a human prostate tissue microarray comprised of tumor-matched normal, BPH, PIN, and tumor spots.

Fig. 3 is a display of representative tissue spots of normal, $\mathrm{BPH}, \mathrm{PIN}$, and tumors of various Gleason grades stained with an anti-arginase II antibody. AII expression was found to be localized to the glandular epithelial region of human prostate tissues. A moderate amount of AII staining was observed in normal, BPH, and PIN tissue spots (Fig. 3A). The tumor group as a whole had the lowest overall AII staining intensity compared to the other histologic categories. However, within the tumor group, low-grade tumors exhibited relatively 


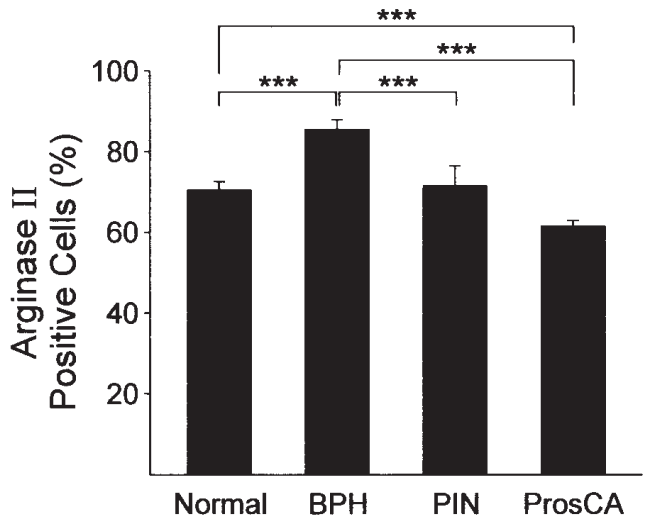

Figure 4. Distribution of arginase II protein expression in the various prostate histological categories. Shown as the percent of AII positive cells $(0-100 \%)$ in normal $(n=251)$, BPH $(n=109)$, PIN $(n=41)$, and ProsCA $(n=598)$ tissue spots. The mean percent of AII positive cells was $70 \%$ in normal, $85 \%$ in $\mathrm{BPH}, 71 \%$ in PIN, and $61 \%$ in ProsCA samples. There were significantly more AII positive cells in BPH tissues compared to normal, PIN, and tumor samples. In addition, AII staining among the normal samples was significantly higher compared to the tumor samples. The vertical lines of the bracket indicate the two prostate histological categories being compared to each other; ${ }^{*} \mathrm{p}<0.05,{ }^{* *} \mathrm{p}<0.01,{ }^{* * *} \mathrm{p}<0.001$.

strong staining with a decline in AII expression as tumors become more undifferentiated, as seen with an intermediategrade tumor as well as with a high-grade tumor (Fig. 3B). It is also important to note that all histologies had variable staining.

We calculated the percentage of target cells (glandular epithelial cells) within each tissue spot that were positively staining for arginase II (0-100\%). Fig. 4 shows the distribution of AII positive cells in the various prostate tissue categories. AII expression was generally lower in prostate cancer than in normal, BPH, or PIN tissues. As a group, the mean percent AII positive cells for BPH $(85 \pm 2.50)$ was the highest, followed by PIN $(71 \pm 4.95)$, then normal $(70 \pm 2.11)$, and the lowest percentage was seen in tumors $(61 \pm 1.49)$. We observed a significant increase in AII staining in BPH tissues compared to normal, PIN, and tumor samples $(\mathrm{p}<0.001)$. In addition, AII staining among the normal samples was significantly higher compared to the tumor samples $(\mathrm{p}<0.001)$.

Arginase II staining across Gleason grades. Although the microarray tumor group as a whole had less AII-positive

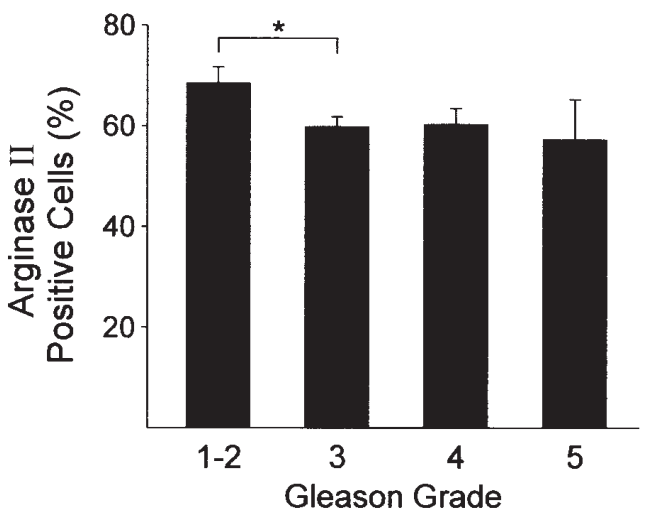

Figure 5. Arginase II expression across the prostate Gleason grades 1-5. Shown as the percent of AII positive cells $(0-100 \%)$ in grade $1-2 \quad(n=97)$, grade $3(n=337)$, grade $4(n=139)$, and grade $5(n=25)$ tissue spots. The mean percent of AII positive cells was $68 \%$ in grade $1-2,60 \%$ in grade 3 , $60 \%$ in grade 4 , and $57 \%$ in grade 5 samples. AII staining in Gleason grade 2 tumor spots was significantly greater than in Gleason grade 3 samples. The vertical lines of the bracket indicate the two Gleason grade categories being compared to each other; ${ }^{*} \mathrm{p}<0.05,{ }^{* *} \mathrm{p}<0.01,{ }^{* * *} \mathrm{p}<0.001$.

cells than normal, BPH, and PIN samples, we did observe a gradient of AII expression across the various prostate cancer tumor grades. Fig. 5 illustrates the percentage of positive cells staining for AII in each of the Gleason grades 2-5 (grades 1 and 2 are combined). AII expression is higher in the lowest grade tumors, but otherwise similar across intermediateto high-grade tumors. The mean percent AII-positive cells for Gleason grade $2(68 \pm 3.20)$ was the highest, followed by Gleason grade $3(60 \pm 2.01)$ and grade $4(60 \pm 3.16)$, with the lowest percentage seen in Gleason grade $5(57 \pm 8.07)$. The percentage of AII staining was significantly greater in the Gleason grade 2 tumor spots compared to Gleason grade 3 samples $(\mathrm{p}<0.05)$. In addition, arginase II is not directly associated with preop PSA, stage, capsular invasion, lymph node positivity, or recurrence status (data not shown).

Polyamine analysis in prostate cancer cell lines. For polyamine analysis, putrescine, spermidine, and spermine levels were measured in all of the prostate cancer cell lines mentioned previously (Table I). The average putrescine $(\mathrm{p}<0.01)$ and spermidine $(\mathrm{p}<0.05)$ levels for LNCaP and LAPC-4 cells were significantly lower than those measured in PC3 and DU145

Table I. Comparison of polyamine levels in human prostate cancer cell lines. ${ }^{a}$

\begin{tabular}{|c|c|c|c|c|}
\hline Cell line & Putrescine & Spermidine & Spermine & Total polyamines \\
\hline LNCaP & $0.82 \pm 0.12$ & $15.21 \pm 3.57$ & $32.61 \pm 5.62$ & $48.63 \pm 8.92$ \\
\hline LAPC-4 & $4.01 \pm 0.22$ & $21.12 \pm 1.08$ & $24.33 \pm 0.83$ & $49.45 \pm 1.89$ \\
\hline PC3 & $14.20 \pm 1.85$ & $31.53 \pm 4.06$ & $24.10 \pm 2.69$ & $69.83 \pm 8.57$ \\
\hline DU145 & $10.58 \pm 1.61$ & $38.10 \pm 4.29$ & $15.45 \pm 0.79$ & $64.13 \pm 6.01$ \\
\hline CL-1 & $27.74 \pm 1.59$ & $28.12 \pm 2.54$ & $26.96 \pm 1.60$ & $82.82 \pm 5.55$ \\
\hline CL-2 & $14.00 \pm 0.92$ & $23.76 \pm 0.89$ & $27.94 \pm 0.68$ & $65.70 \pm 1.56$ \\
\hline
\end{tabular}

aPolyamine levels expressed as nmoles/mg of protein \pm SD. 
cells. Spermine levels in the LNCaP cells were significantly increased as compared to LAPC-4, PC3, and DU145 $(\mathrm{p}<0.05)$. Although the spermine levels for LAPC-4 cells were significantly greater than DU145 cells $(\mathrm{p}<0.001)$, they were not significantly different compared to PC3 cells. Additionally, we compared polyamine levels of LNCaP cells to CL-1 and CL-2, and once again noted that the LNCaPderived androgen-independent clones were behaving more similarly to PC3 and DU145 with respect to polyamine measurements than to the original LNCaP cell line. Putrescine $(\mathrm{p}<0.001)$ and spermidine $(\mathrm{p}<0.01)$ levels were significantly lower in LNCaP cells compared to both CL-1 and CL-2 cells. There was no appreciable change in spermine levels detected among the three cell lines.

Overall, LNCaP and LAPC-4 cells, which have the highest AII expression, exhibited decreased putrescine and spermidine levels. On the other hand, PC3, DU145, CL-1, and CL-2 cells have low levels of AII expression and show an opposite trend for polyamine production. Similarly, total polyamines were significantly lower in the androgen-dependent cell lines than those measured in the androgen-independent cell lines $(\mathrm{p}<0.01)$.

\section{Discussion}

Arginase II expression pattern in prostate cancer cell lines. Previous reports have suggested that arginase activity is elevated in prostatic carcinomas compared to benign tissues (30-32). Our extensive study of AII expression in prostate cancer specimens revealed differential expression of this enzyme. Based on initial cell culture studies, we demonstrated that arginase II expression was most prominent in the more differentiated prostate cancer cell lines (LNCaP, LAPC-4) and less prominent in undifferentiated, androgen-independent cell lines (PC3, DU145). To further investigate the issue of AII and androgen susceptibility, we extended our study to include two new aggressive LNCaP-derived androgen-independent cell lines, CL-1 and CL-2 (22). We demonstrated loss of AII in the androgen-independent cell lines (CL-1, CL-2) compared to the original LNCaP cell line. Collectively, these results confirm that AII is differentially expressed in androgendependent versus androgen-independent human prostate cancer cell lines. It appears that the loss of AII expression in the androgen-independent cell line, CL-1, is irreversible since AII remains absent even when the cells are returned to androgen containing medium as seen with the CL-2 cell line. The decreased levels of AII among the androgen-independent cell lines, PC3, DU145, CL-1, and CL-2, suggests that low levels of AII may be an indicator of more aggressive, late-stage prostate cancer and could be contributing to androgenindependent progression. This brings up the question of whether the arginase II gene is being regulated by the presence of androgens in the prostate.

Regulation of arginase by androgens. In other studies, it has been shown that after administration of testosterone to castrated rats, arginase activity is enhanced, thus implying prostatic arginase activity is hormone-dependent $(33,34)$. Furthermore, in kidneys of female mice, the addition of testosterone has led to induction of ODC and AII and an associated reduction in OAT levels $(35,36)$. As expected, castration of male mice has shown the opposite effect with a decline in levels of ODC and AII and a simultaneous increase in OAT $(36,37)$. Consistent with these studies, we found ODC and even more dramatically AII expression to be significantly higher in the androgen-dependent prostate cancer cell lines compared to the androgen-independent lines. In contrast, OAT levels were reduced in the androgen-dependent cell lines and enhanced in the androgen-independent cells. Moreover, the AII and OAT expression profiles seen in the CL-1 and CL-2 cell lines were more similar to those observed in PC3 and DU145 than to the original cell line they were derived from, LNCaP, making these findings even more striking. This suggests that during prostate cancer progression, AII expression diminishes, while OAT expression is enhanced. Although ODC RNA levels are slightly greater in the androgen-dependent cell lines compared to the androgenindependent cell lines, it is difficult to draw any significant conclusions from these particular data due to the rapid rate of metabolism of this enzyme leading to overall low levels.

Arginase II expression in benign and malignant prostate tissue. We wanted to broaden our study to include a large number of normal and malignant prostatic samples and thus we turned to a prostate tissue microarray. From our analysis, we found the highest percentage of AII positive cells in the $\mathrm{BPH}$ samples, followed by PIN and normal tissues, with the lowest expression levels generally seen in prostate cancer. Similarly, a report by Elgun et al found significantly lower levels of serum arginase activity in prostatic cancer compared to normal and BPH groups. In addition, the BPH group had significantly higher levels of arginase than the control group (19).

The majority of literature concerning AII and prostate cancer is based on tissue or serum enzyme activity rather than gene expression. Our study is the first high-throughput, parallel analysis of arginase II expression in normal, BPH, PIN, and prostate tumor samples using an anti-arginase II antibody. There is some discrepancy between previous studies on arginase activity (29-31) and our current study on arginase expression in prostate tissues; however, technical strategies and sample size could possibly explain these differences. These other studies may be enriched with highor low-expressing cases and only with many cases can one be assured of the staining spectrum.

Although we had anticipated the tumor group as a whole to show the greatest AII expression of the various histological categories, this discrepancy could be explained by the AII staining intensity we observed across the Gleason grades within the tumor group. We detected significantly stronger AII expression in the lowest grade tumors with a decline in expression as the tumors became less differentiated. Several studies support our finding that arginase activity is inversely correlated with the histological grade of prostate tumors $(19,31,38)$. Since AII expression is localized to the glandular epithelial regions of the prostate, it seems reasonable to hypothesize that as tumors become less differentiated and lose their glandular structure, AII expression diminishes. Therefore, the AII expression gradient across tumor grades, and most importantly the low levels of AII seen in the 
medium- to high-grade tumors, may account for the tumor group having the lowest percentage of AII positive cells.

Any increase in AII expression in the low-grade tumors above the non-malignant tissue levels may be masked by the lower expression observed in high-grade tumors. Arginase II expression among prostate cancer patients did not directly correlate with preop PSA, stage, capsular invasion, lymph node positivity, or recurrence status, suggesting that arginase II could possibly be used as an independent prognostic marker. Specifically, whereas PSA cannot by itself distinguish those patients with good vs. poor prognosis vs. those who will do well even without treatment, arginase II expression - and its subsequent disappearance - could potentially be used as a warning marker to identify those patients at higher risk.

Involvement of arginase II in polyamine synthesis. The notion that arginase II is a key regulator of prostate physiology is strongly supported by its involvement in polyamine biosynthesis $(10,14,39,40)$. AII functions to produce the ornithine necessary for synthesis of polyamines (putrescine, spermidine, and spermine), which are important for normal and neoplastic cell proliferation $(6,41)$. Our analysis of polyamines in the various prostate cancer cell lines supports a possible correlation between arginase II expression and polyamine levels. More specifically, the androgen-dependent prostate cancer cell lines, which have the greatest expression of AII, have statistically lower levels of putrescine and spermidine than the androgen-independent cell lines. On the contrary, the less differentiated androgen-independent cell lines have the highest expression of OAT and statistically higher levels of putrescine and spermidine than the more differentiated cell lines. This is consistent with previous implications that decreased spermidine levels are characteristic of a higher degree of differentiation, as seen in LNCaP and LAPC-4 cell lines. The more aggressive androgen-independent prostate cancer cell lines, PC3, DU145, CL-1, and CL-2, showed increased putrescine and spermidine levels, which are required for prostatic cell proliferation (42).

Interaction between arginase II and ornithine aminotransferase. Alternate enzymes acting in the arginine metabolic and polyamine synthetic pathways may be upregulated in late stage prostate cancer and compensate for the lack of AII expression. This phenomenon has been shown in mice where OAT produces the main source of ornithine in the GI tract necessary for survival of the neonatal mouse $(43,44)$. At two weeks of age, OAT levels drop dramatically in mice, while at the same time arginase levels increase and become the main producer of ornithine (44). These findings support the possibility that an alternate pathway exists in prostate cancer to compensate for loss of AII expression.

The inverse relationship we observed between AII and OAT seems to correlate with the polyamine profile seen in the prostate cancer cell lines. This result suggests that early stage prostate cancer uses the arginase pathway to produce the ornithine necessary for polyamine production. In contrast, late stage prostate carcinoma, which has even higher proliferative demands, may generate polyamines from the ornithine derived from OAT. The colocalization of AII and OAT in the mitochondria and their shared function in the biosynthesis and utilization of ornithine could logically support the proposed compensatory mechanism between these two enzymes during prostate cancer progression $(45,46)$. The shift in polyamine production in these cell lines may involve changes in the polyamine biosynthetic enzymes in addition to the supply of ornithine. Antizyme and S-adenosylmethionine decarboxylase (AdoMetDC) are two important biosynthetic enzymes responsible for regulating polyamine levels. Antizyme is involved in the feedback regulation of polyamine levels through its ability to cause degradation of ODC, while AdoMetDC acts as the aminopropyl donor to synthesize spermidine and spermine from putrescine $(47,48)$. In our studies, both enzymes showed increased mRNA levels in the androgen-dependent cell lines with high AII present, which could explain the reduction seen in putrescine and spermidine levels in these cell lines (data not shown).

In conclusion, this study is the first thorough analysis of several enzymes in the arginine metabolic pathway with respect to prostate cancer development. Our results showing elevated AII expression in androgen-dependent cell lines and in more differentiated prostate tissues suggest that high AII may be a useful marker of early stage prostatic adenocarcinoma. Currently, we are in the process of determining whether the differential expression of arginase II observed in prostate cancer is driven by androgen susceptibility and/or if it is an indicator of degree of differentiation. The loss of AII expression observed in the LNCaP-derived androgenindependent cell line, CL-1, suggests that AII expression is tied to androgen dependence/independence and may be a useful marker for therapy stratification.

It appears that the increase in OAT expression within androgen-independent prostate cancer cell lines serves to compensate for the loss of AII and in turn synthesize the ornithine necessary for polyamine production. To the best of our knowledge, the present study provides the first detailed description of OAT expression in prostate cancer. Our findings suggest a more complex interrelationship between arginase and other enzymes in the arginine metabolic pathway, such as OAT, ODC, and polyamine biosynthetic enzymes. Future studies including regulatory analysis are needed to address these mechanistic possibilities.

\section{Acknowledgements}

We wish to acknowledge Suzie Kuhn for her technical assistance with the HPLC analysis; and Lee Goodglick and Chia-Ling Gau for their suggestions on the manuscript. We would also like to thank the UCLA Tissue Procurement Core Laboratory (TPCL), which is partially supported by an NCI grant (CA16042), for providing us the necessary prostate tissues to conduct some of our studies. This work was supported by grants from the DOD/US Army DAMD 17-031-0201 and the UCLA SPORE in Prostate Cancer NCI CA92131 to W.W.G.

\section{References}

1. Jemal A, Siegel R, Ward E, Murray T, Xu J, Smigal C and Thun MJ: Cancer statistics, 2006. CA Cancer J Clin 56: 106-130, 2006.

2. Bunting PS: Screening for prostate cancer with prostate-specific antigen: beware the biases. Clin Chim Acta 315: 71-97, 2002. 
3. Tabib A and Bachrach U: Role of polyamines in mediating malignant transformation and oncogene expression. Int $\mathbf{J}$ Biochem Cell Biol 31: 1289-1295, 1999.

4. Calandra RS, Rulli SB, Frungieri MB, Suescun MO and Gonzalez-Calvar SI: Polyamines in the male reproductive system. Acta Physiol Pharmacol Ther Latinoam 46: 209-222, 1996.

5. Pegg AE: Polyamine metabolism and its importance in neoplastic growth and a target for chemotherapy. Cancer Res 48: 759-774, 1988.

6. Russell DH: Polyamines and prostatic function. Prog Clin Biol Res 75A: 207-224, 1981.

7. Bettuzzi S, Davalli P, Astancolle S, Carani C, Madeo B, Tampieri A and Corti A: Tumor progression is accompanied by significant changes in the levels of expression of polyamine metabolism regulatory genes and clusterin (sulfated glycoprotein 2) in human prostate cancer specimens. Cancer Res 60: 28-34, 2000.

8. Seiler N, Atanassov CL and Raul F: Polyamine metabolism as target for cancer chemoprevention (Review). Int J Oncol 13: 993-1006, 1998.

9. Pegg AE, Shantz LM and Coleman CS: Ornithine decarboxylase: structure, function and translational regulation. Biochem Soc Trans 22: 846-852, 1994.

10. Iyer R, Jenkinson CP, Vockley JG, Kern RM, Grody WW and Cederbaum S: The human arginases and arginase deficiency. J Inherit Metab Dis 21 (Suppl 1): 86-100, 1998.

11. Mezl VA and Knox WE: Metabolism of arginine in lactating rat mammary gland. Biochem J 166: 105-113, 1977.

12. Yoneda Y, Roberts E and Dietz GW Jr: A new synaptosomal biosynthetic pathway of glutamate and GABA from ornithine and its negative feedback inhibition by GABA. J Neurochem 38: 1686-1694, 1982.

13. Jenkinson CP, Grody WW and Cederbaum SD: Comparative properties of arginases. Comp Biochem Physiol B Biochem Mol Biol 114: 107-132, 1996.

14. Cederbaum SD, Yu H, Grody WW, Kern RM, Yoo P and Iyer RK: Arginases I and II: do their functions overlap? Mol Genet Metab 81 (Suppl 1): 38-44, 2004.

15. Marton LJ and Pegg AE: Polyamines as targets for therapeutic intervention. Annu Rev Pharmacol Toxicol 35: 55-91, 1995.

16. Mohan RR, Challa A, Gupta S, Bostwick DG, Ahmad N, Agarwal R, Marengo SR, Amini SB, Paras F, MacLennan GT, Resnick MI and Mukhtar H: Overexpression of ornithine decarboxylase in prostate cancer and prostatic fluid in humans. Clin Cancer Res 5: 143-147, 1999.

17. Vockley JG, Jenkinson CP, Shukla H, Kern RM, Grody WW and Cederbaum SD: Cloning and characterization of the human type II arginase gene. Genomics 38: 118-123, 1996.

18. Chang CI, Liao JC and Kuo L: Macrophage arginase promotes tumor cell growth and suppresses nitric oxide-mediated tumor cytotoxicity. Cancer Res 61: 1100-1106, 2001.

19. Elgun S, Keskinege A, Yilmaz E, Baltaci S and Beduk Y: Evaluation of serum arginase activity in benign prostatic hypertrophy and prostatic cancer. Int Urol Nephrol 31: 95-99, 1999.

20. Singh R, Pervin S, Karimi A, Cederbaum S and Chaudhuri G: Arginase activity in human breast cancer cell lines: N(omega)hydroxy-L-arginine selectively inhibits cell proliferation and induces apoptosis in MDA-MB-468 cells. Cancer Res 60: 3305-3312, 2000.

21. Kononen J, Bubendorf L, Kallioniemi A, Barlund M, Schraml P, Leighton S, Torhorst J, Mihatsch MJ, Sauter G and Kallioniemi OP: Tissue microarrays for high-throughput molecular profiling of tumor specimens. Nat Med 4: 844-847, 1998.

22. Freedland SJ, Seligson DB, Liu AY, Pantuck AJ, Paik SH, Horvath S, Wieder JA, Zisman A, Nguyen D, Tso CL, Palotie AV and Belldegrun AS: Loss of CD10 (neutral endopeptidase) is a frequent and early event in human prostate cancer. Prostate 55: 71-80, 2003.

23. Pegg AE, Wechter R, Pakala R amd Bergeron RJ: Effect of N1,N12-bis(ethyl)spermine and related compounds on growth and polyamine acetylation, content, and excretion in human colon tumor cells. J Biol Chem 264: 11744-11749, 1989.

24. Seligson D, Horvath S, Huerta-Yepez S, Hanna S, Garban H, Roberts A, Shi T, Liu X, Chia D, Goodglick L and Bonavida B: Expression of transcription factor Yin Yang 1 in prostate cancer. Int J Oncol 27: 131-141, 2005.
25. Humphrey PA: Gleason grading and prognostic factors in carcinoma of the prostate. Mod Pathol 17: 292-306, 2004.

26. Han KR, Seligson DB, Liu X, Horvath S, Shintaku PI, Thomas GV, Said JW and Reiter RE: Prostate stem cell antigen expression is associated with gleason score, seminal vesicle invasion and capsular invasion in prostate cancer. J Urol 171: $1117-1121,2004$.

27. Horoszewicz JS, Leong SS, Kawinski E, Karr JP, Rosenthal H, Chu TM, Mirand EA and Murphy GP: LNCaP model of human prostatic carcinoma. Cancer Res 43: 1809-1818, 1983.

28. van Bokhoven A, Varella-Garcia M, Korch C, Johannes WU, Smith EE, Miller HL, Nordeen SK, Miller GJ and Lucia MS: Molecular characterization of human prostate carcinoma cell lines. Prostate 57: 205-225, 2003.

29. Bronte V, Kasic T, Gri G, Gallana K, Borsellino G, Marigo I, Battistini L, Iafrate M, Prayer-Galetti T, Pagano F and Viola A: Boosting antitumor responses of $\mathrm{T}$ lymphocytes infiltrating human prostate cancers. J Exp Med 201: 1257-1268, 2005.

30. Harris BE, Pretlow TP, Bradley EL Jr, Whitehurst GB and Pretlow TG II: Arginase activity in prostatic tissue of patients with benign prostatic hyperplasia and prostatic carcinoma. Cancer Res 43: 3008-3012, 1983.

31. Keskinege A, Elgun S and Yilmaz E: Possible implications of arginase and diamine oxidase in prostatic carcinoma. Cancer Detect Prev 25: 76-79, 2001.

32. Yamanaka H, Mayuzumi T, Matsuoka M, Shimazaki J and Shida K: Arginase in human urogenital tumors. GANN 63: 693-700, 1972 .

33. Mendez JD, Sosa A and Palomar-Morales M: Effect of L-arginine on arginase activity in male accessory sex glands of alloxantreated rats. Reprod Toxicol 16: 809-813, 2002.

34. Yamanaka H, Kirdani RY, Saroff J, Murphy GP and Sandberg AA: Effects of testosterone and prolactin on rat prostatic weight, 5alpha-reductase, and arginase. Am J Physiol 229: 1102-1109, 1975.

35. Manteuffel-Cymborowska M, Chmurzynska W, Peska M and Grzelakowska-Sztabert B: Arginine and ornithine metabolizing enzymes in testosterone-induced hypertrophic mouse kidney. Int J Biochem Cell Biol 27: 287-295, 1995.

36. Levillain O, Diaz JJ, Blanchard O and Dechaud H: Testosterone down-regulates ornithine aminotransferase gene and up-regulates arginase II and ornithine decarboxylase genes for polyamines synthesis in the murine kidney. Endocrinology 146: 950-959, 2005.

37. Rui H, Brekke I, Morkas L and Purvis K: Androgen interaction with the polyamine system of the rat prostate. Andrologia 19: 134-142, 1987.

38. Pretlow TG II, Harris BE, Bradley EL Jr, Bueschen AJ, Lloyd KL and Pretlow TP: Enzyme activities in prostatic carcinoma related to Gleason grades. Cancer Res 45: 442-446, 1985.

39. Holtta E and Pohjanpelto P: Polyamine dependence of Chinese hamster ovary cells in serum-free culture is due to deficient arginase activity. Biochim Biophys Acta 721: 321-327, 1982.

40. Oka T and Perry JW: Arginase affects lactogenesis through its influence on the biosynthesis of spermidine. Nature 250: 660-661, 1974.

41. Pegg AE and McCann PP: Polyamine metabolism and function. Am J Physiol 243: C212-C221, 1982.

42. Schipper RG, Romijn JC, Cuijpers VM and Verhofstad AA: Polyamines and prostatic cancer. Biochem Soc Trans 31: 375-380, 2003.

43. Peraino C, Bunville LG and Tahmisian TN: Chemical, physical, and morphological properties of ornithine aminotransferase from rat liver. J Biol Chem 244: 2241-2249, 1969.

44. Wang T, Lawler AM, Steel G, Sipila I, Milam AH and Valle D: Mice lacking ornithine-delta-aminotransferase have paradoxical neonatal hypoornithinaemia and retinal degeneration. Nat Genet 11: 185-190, 1995.

45. Mitchell GA, Looney JE, Brody LC, Steel G, Suchanek M, Engelhardt JF, Willard HF and Valle D: Human ornithine-deltaaminotransferase. cDNA cloning and analysis of the structural gene. J Biol Chem 263: 14288-14295, 1988.

46. Zintz CB and Inana G: Analysis of the human ornithine aminotransferase gene family. Exp Eye Res 50: 759-770, 1990.

47. Hayashi T, Matsufuji S and Hayashi S: Characterization of the human antizyme gene. Gene 203: 131-139, 1997.

48. Kramer DL, Sufrin JR and Porter CW: Modulation of polyaminebiosynthetic activity by $\mathrm{S}$-adenosylmethionine depletion. Biochem J 249: 581-586, 1988. 